\title{
Impact of Concurrent Transmissions on Downstream Throughput in Multi-hop Cellular Networks
}

\author{
Jaeweon Cho \\ Samsung Electronics Co., Ltd. \\ Telecommunication R\&D Center \\ 416 Maetan-3, Suwon, Gyeonggi, 442-600, Korea \\ Email: jaeweon.cho@ieee.org
}

\author{
Zygmunt J. Haas \\ Cornell University \\ School of Electrical and Computer Engineering \\ Ithaca, New York 14853, USA \\ Email: haas@ece.cornell.edu
}

\begin{abstract}
In this paper, we investigate the impacts of transmission concurrency on the throughput of the downstream channel in multi-hop cellular networks. In particular, this paper focuses on the tradeoff between the improvement of resource reuse and the increase in radio interference. We evaluate and compare the throughput for various concurrency types of multihop relaying. We also propose a hybrid control strategy, in which we advocate the use of both, the concurrent and the nonconcurrent transmissions. Our results show that the throughput can be increased by allowing transmission concurrency, but the concurrency does not always result in higher throughput, relative to the non-concurrency case, because of the interference between concurrently transmitting links. In the random topology with shadowing, depending on the particular circumstances, either the concurrent or the non-concurrent case can be the optimal transmission strategy. Thus, in order to achieve a significant improvement, a hybrid scheme should be employed. Additionally, if concurrency is applied, it should be used among the different downstream paths, rather than between the hops on the same downstream path.
\end{abstract}

\section{INTRODUCTION}

Multi-hop cellular networks have been proposed as an extension to the conventional single-hop cellular network by combining the fixed cellular infrastructure with the multi-hop relaying technology, usually used in ad-hoc networks. Through the use of the multi-hop relaying, more favorable path than a direct single-hop link can be utilized between a base station (BS) and a mobile station (MS), so that the system capacity can be considerably enhanced [1].

One way to increase the capacity of the multi-hop systems is to allow concurrency among the multi-hop transmissions. Such concurrency can improve the channel reuse efficiency, but also leads to an increase in the interference. Toumpis and Goldsmith [2] showed that the concurrent transmission can enhance the system capacity in multi-hop cellular networks. However, their results were obtained for a single cell system and also for just two cases of network topology, i.e. a linear topology and a single realization of a random topology. Hence, those results are insufficient to demonstrate, in general,

The work of Jaeweon Cho was supported in part by Samsung Electronics Co., Ltd.

The work of Zygmunt J. Haas was supported in part by the DoD Multidisciplinary University Research Initiative (MURI) programs administered by the ONR under contract number N00014-00-1-0564 and by the AFOSR under contract number F49620-02-1-0217, and by the National Science Foundation under grant numbers ANI-9980521 and ANI-0081357. the concurrency tradeoff between the interference and the channel reuse efficiency. Moreover, several studies reported that it is not easy to enhance the capacity of code division multiple access (CDMA) systems by the use of the multihop relaying [3]-[7]. This is mainly due to the interference increase resulting from by the concurrent transmissions. Since such interference might be the most significant factor limiting the network capacity, the concurrent transmission should be comprehensively studied and cautiously applied.

In this paper, we investigate the impact of concurrent transmissions on the throughput in the multi-hop cellular networks. The concurrency of interest in this work is classified into two types: The concurrency among the different hops along the path to one target MS, which is referred to as type A concurrency; the other type is the concurrency among the hops on the different downstream paths for multiple target MS$\mathrm{s}$, which is referred to as type $B$ concurrency. Our previous work [1] showed that the type A concurrency can increase the throughput, but such an increase is rather small. In this paper, we extend our investigation to the type B concurrency, and compare the performance of these two concurrency types. We also propose a hybrid control scheme in which both, the concurrent and the non-concurrent transmissions, can be used, depending on the particular circumstances.

This paper focuses on the best-effort (delay-tolerant) type of service in the downstream direction, from a BS to a target MS. Since the nature of data traffic for most services on the Internet is asymmetric, efficient utilization of resource on the downlink is becoming more and more important. For this reason, recently standardized systems, which is evolved from the third-generation $(3 \mathrm{G})$ mobile communication systems, aim at supporting high data rate packet services on the downlink, e.g., the 1xEV-DO system [8] and the HSDPA system [9]. In the next section, we describe our system model. The throughput gain for each concurrency type is derived in Section III, while the numerical results are presented and compared in Section IV. In Section V, we discuss the impact of the concurrency on the capacity of the conventional power-controlled CDMA systems. Finally, we draw some conclusions in Section VI.

\section{SySTEM MOdEL}

Let us consider a cellular system where a number of BS-s are placed according to a hexagonal grid pattern. Every MS in 
the system is assumed to be capable of multi-hop relaying. We also assume a fully loaded system and an infinite buffers at the network nodes. The upstream and the downstream channels are assumed to work independently. The downstream transmission may involve multiple relaying MS-s, in which case packets are relayed between neighboring MS-s on the same downstream path through the use of time division duplex (TDD), as in [10]. The channels assigned to each cell share the same frequency spectrum by CDMA. Time division multiplexing (TDM) is selected for multiplexing downstream channels at the BS; i.e., each BS provides one downstream channel to one target MS at a time. ${ }^{1}$

In our system, rate control is utilized based on the channel condition, as in the $1 \mathrm{xEV}-\mathrm{DO}$ system and the HSDPA systems. $^{2}$ The transmission power $P$ is fixed, and the data rate assigned to each link $R$ is determined by the signal to interference and noise ratio (SINR). Shannon capacity formula is then used in the rate control model to evaluate the network capacity [2]:

$$
R=W \log _{2}(1+\mathrm{SINR}),
$$

where $W$ is the channel bandwidth and SINR at the receiver of the transmission from node $i$ is given by:

$$
\mathrm{SINR}=\frac{L_{i} P_{i}}{\sum_{j \in \mathcal{J}, j \neq i} L_{j} P_{j}},
$$

where the subscript $j$ denotes the a transmitting node (BS or MS) and $\mathcal{J}$ is the set of transmitting nodes at a given time. In this paper, the interference limited scenario is considered, and so the contribution of the thermal noise to the SINR is neglected. In (2), $L_{i}$ denotes the link gain, so that at distance $d_{i}$ from the node $i$, it can be represented as:

$$
L_{i}=A \cdot d_{i}^{-\gamma} \cdot 10^{\zeta_{i} / 10},
$$

where $A$ is a constant, $\gamma$ is the path loss exponent, and $\zeta_{i}$ is the shadowing random variable.

\section{Throughput GAIN}

The downstream throughput can be calculated as the effective data rate on the direct link or on the multi-hop path between BS and the target MS. We define the decision parameter and the throughput gain based on this effective data rate.

\section{A. Simple Relaying without Concurrency}

We first consider the case of simple relaying, in which only one downstream channel for a target MS is provided in each cell at any time. Let us assume that a time period for data transmission $T$ is assigned to the target MS. In the conventional system, the effective data rate is identical to the

\footnotetext{
${ }^{1}$ In multi-rate CDMA systems, packet traffic channels can be assigned in a code division multiplexing (CDM) or TDM fashion; e.g., TDM is used in the 1xEV-DO system, and TDM and/or CDM in HSDPA system.

${ }^{2}$ For the best effort type data services, the rate control is preferable to the power control, because in the rate control scheme high SINR available over a large portion of the cell area can be exploited to provide higher data rate by the rate control.
}

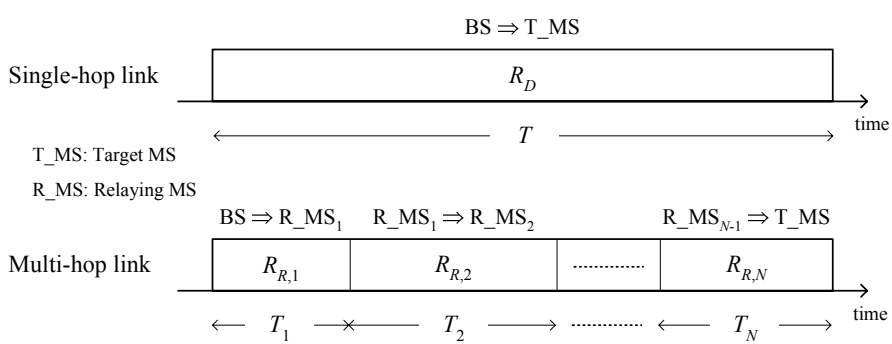

Fig. 1. Transmission data rates in single-hop and multi-hop transmission

data rate of the direct single-hop link, $R_{D}$. Let $U_{R}$ be the effective data rate of the relaying path comprised of multihop links. Each hop is assigned the downstream channel for a short time duration, as shown in Fig. 1. We assume that the relaying MS-s act as forwarders only during $T$. Hence the transmitted amount of data for each time slot should be identical for all the nodes on the relaying path $^{3}$; i.e.,

$$
T_{1} R_{R, 1}=T_{2} R_{R, 2}=\cdots=T_{N} R_{R, N}
$$

where $R_{R, n}$ denotes the data rate on the $n$-th hop of the relaying path $(n=1, \cdots, N)$, and $N$ is the number of hops. Then $U_{R}$ can be given by the number of effective transmitted bits per $T$, and the decision parameter $D$ can be defined as the ratio of the two effective data rates, the effective data rate in the multi-hop system to the effective date rate in the conventional system [1]:

$$
D \equiv \frac{U_{R}}{R_{D}}=\frac{T_{N} R_{R, N}}{T} \cdot \frac{1}{R_{D}}=\frac{1}{\sum_{n=1}^{N} R_{D} / R_{R, n}} .
$$

Note that in our network model, either the direct link or the multi-hop path can be selected, whichever case provides higher throughput. That is, the multi-hop path is selected if $D>1$; otherwise, the direct link is selected. Then the throughput gain, $G$, in a network that uses such a hybrid scheme can be defined as the ratio of throughput of the most favorable case (the direct link or the multi-hop path) to that of the direct link. Therefore,

$$
G \equiv\left\{\begin{array}{ll}
D & \text { if } D>1 \\
1 & \text { otherwise }
\end{array} .\right.
$$

\section{B. Type A Concurrency}

In the case of the type A concurrency, while only one target MS is provided with a downstream channel at a time, the concurrent transmissions on this channel are allowed on the different hops of the path. Each concurrent transmission uses the distinct spreading code. Note that even though the concurrency occurs, all the transmitted data during $T$ is for one target mobile MS. Hence, the transmitted amount of data in each time slot is limited by the hop whose data rate is the minimum among the multiple concurrent hops. It also should

\footnotetext{
${ }^{3}$ In this paper the time slot means a logical time slot which has a variable length.
} 


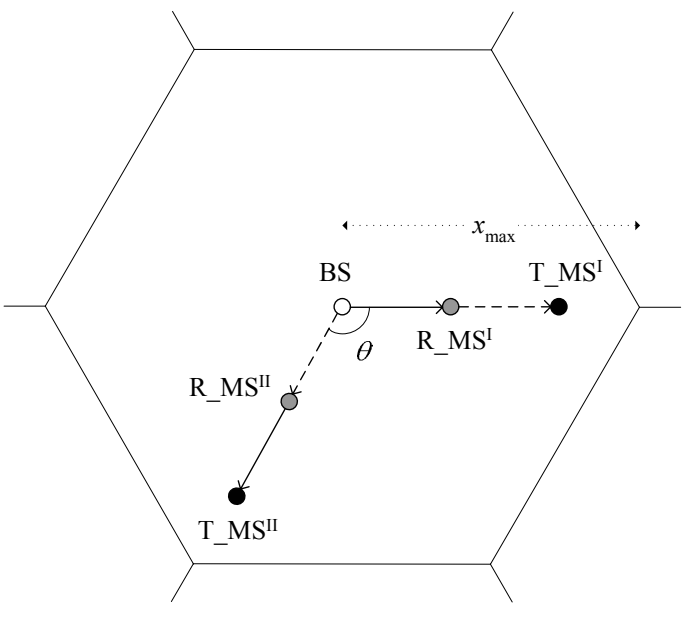

(a) Topology
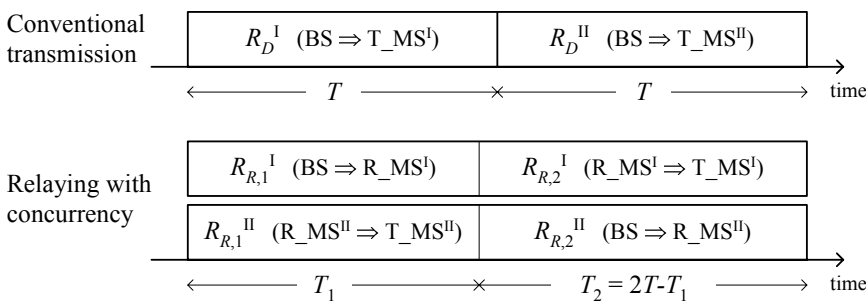

(b) Transmission timing

Fig. 2. Illustration of the type B concurrency.

be equal to the transmitted amount of data in the other time slots. So, as in (4),

$$
T_{1} \cdot \min _{n \in \mathcal{T}_{1}}\left\{R_{R, n}\right\}=\cdots=T_{M} \cdot \min _{n \in \mathcal{T}_{M}}\left\{R_{R, n}\right\},
$$

where $\mathcal{T}_{m}$ is the set of the hops sharing the $m$-th time slot, and $M(2 \leq M \leq N)$ is the number of time slots during $T$. Then $U_{R}$ can be represented by:

$$
U_{R}=\frac{1}{1 / \min _{n \in \mathcal{T}_{1}}\left\{R_{R, n}\right\}+\cdots+1 / \min _{n \in \mathcal{T}_{M}}\left\{R_{R, n}\right\}} .
$$

We use the same selection criterion for the multi-hop path as applied in the case of the simple relaying and the same definition for of the metrics; i.e., $D \equiv U_{R} / R_{D}$ and $G \equiv$ $\max \{D, 1\}$.

\section{Type B Concurrency}

With the type B concurrency, the different downstream paths for multiple target MS-s can share time slots. For simplicity, we consider two downstream paths (for two target MS-s) with 2-hop paths.

The multi-hop relaying with the type $B$ concurrency is illustrated in Fig. 2, where the superscript I and II denote each target MS. Since the relaying MS-s act as forwarders only during $2 T$, the transmitted amounts during $T_{1}$ and $T_{2}$ should be the same, i.e., $T_{1} R_{R, 1}^{\mathrm{I}}=T_{2} R_{R, 2}^{\mathrm{I}}$ and $T_{1} R_{R, 1}^{\mathrm{II}}=T_{2} R_{R, 2}^{\mathrm{II}}$. However, $R_{R, 1}^{\mathrm{I}} / R_{R, 2}^{\mathrm{I}}$ is generally not equal to $R_{R, 1}^{\mathrm{II}} / R_{R, 2}^{\mathrm{II}}$. Thus the two equations cannot be satisfied simultaneously with the same time division of the two paths. Rather, we should assign different time division to each one of the two paths, so that for both channels the transmitted amounts on the first hop is equal to that on the second hop.

We illustrate the transmission timing with the different time division of each path, in Fig. 3. According to the length of $T^{\mathrm{I}}$ and $T^{\mathrm{II}}$, the applied time division is divided into 2 cases. We first consider the case of $T^{\mathrm{I}} \leq T^{\mathrm{II}}$. From Fig. 3 (a), we can formulate the following set of equations:

$$
\begin{aligned}
& R_{R, 1}^{\mathrm{I}} T^{\mathrm{I}}=\left(T^{\mathrm{II}}-T^{\mathrm{I}}\right) R_{R, 2}^{\mathrm{I}}+\left(2 T-T^{\mathrm{II}}\right) R_{R, 3}^{\mathrm{I}}, \\
& R_{R, 1}^{\mathrm{II}} T^{\mathrm{I}}+\left(T^{\mathrm{II}}-T^{\mathrm{I}}\right) R_{R, 2}^{\mathrm{II}}=\left(2 T-T^{\mathrm{II}}\right) R_{R, 3}^{\mathrm{I}} .
\end{aligned}
$$

Solving the these two equations for $T^{\mathrm{I}}$ and $T^{\mathrm{II}}$, and we obtain:

$$
\begin{aligned}
T^{\mathrm{I}} & =\frac{\left(R_{R, 2}^{\mathrm{II}} R_{R, 3}^{\mathrm{I}}+R_{R, 2}^{\mathrm{I}} R_{R, 3}^{\mathrm{II}}\right) 2 T}{C_{a}}, \\
T^{\mathrm{II}} & =\frac{\left[\left(R_{R, 2}^{\mathrm{II}}-R_{R, 1}^{\mathrm{II}}\right) R_{R, 3}^{I}+\left(R_{R, 2}^{\mathrm{I}}-R_{R, 1}^{\mathrm{I}}\right) R_{R, 3}^{\mathrm{II}}\right] 2 T}{C_{a}},
\end{aligned}
$$

where

$$
\begin{aligned}
C_{a} \equiv\left(R_{R, 1}^{\mathrm{I}}+R_{R, 2}^{\mathrm{I}}\right)\left(R_{R, 2}^{\mathrm{II}}+R_{R, 3}^{\mathrm{II}}\right) & \\
& -\left(R_{R, 3}^{\mathrm{I}}-R_{R, 2}^{\mathrm{I}}\right)\left(R_{R, 1}^{\mathrm{II}}-R_{R, 2}^{\mathrm{II}}\right) .
\end{aligned}
$$

Hence, the effective data rates during $2 T$ for the two multi-hop paths are given by:

$$
\begin{aligned}
U_{R}^{\mathrm{I}} & =\frac{T^{\mathrm{I}} R_{R, 1}^{\mathrm{I}}}{2 T}=\frac{\left(R_{R, 3}^{\mathrm{I}} R_{R, 2}^{\mathrm{II}}+R_{R, 2}^{\mathrm{I}} R_{R, 3}^{\mathrm{II}}\right) R_{R, 1}^{\mathrm{I}}}{C_{a}} \\
U_{R}^{\mathrm{II}} & =\frac{\left(2 T-T^{\mathrm{II}}\right) R_{R, 3}^{\mathrm{II}}}{2 T}=\frac{\left(R_{R, 1}^{\mathrm{I}} R_{R, 2}^{\mathrm{II}}+R_{R, 2}^{\mathrm{I}} R_{R, 1}^{\mathrm{II}}\right) R_{R, 3}^{\mathrm{II}}}{C_{a}} .
\end{aligned}
$$

Now let us proceed with the case of $T^{\mathrm{I}}>T^{\mathrm{II}}$, which is illustrated in Fig. 3 (b). As in (9) and (10), by formulating a set of equations, we obtain the following solution:

$$
\begin{aligned}
T^{\mathrm{I}} & =\frac{\left[\left(R_{R, 1}^{\mathrm{II}}+R_{R, 2}^{\mathrm{II}}\right) R_{R, 3}^{\mathrm{I}}-\left(R_{R, 1}^{\mathrm{I}}-R_{R, 2}^{\mathrm{I}}\right) R_{R, 3}^{\mathrm{II}}\right] 2 T}{C_{b}} \\
T^{\mathrm{II}} & =\frac{\left(R_{R, 3}^{\mathrm{I}} R_{R, 2}^{\mathrm{II}}+R_{R, 3}^{\mathrm{II}} R_{R, 2}^{\mathrm{I}}\right) 2 T}{C_{b}}
\end{aligned}
$$

where

$$
\begin{aligned}
C_{b} \equiv\left(R_{R, 1}^{\mathrm{II}}+R_{R, 3}^{\mathrm{II}}\right) R_{R, 2}^{\mathrm{I}}+\left(R_{R, 1}^{\mathrm{I}}+R_{R, 3}^{\mathrm{I}}\right) R_{R, 2}^{\mathrm{II}} \\
+2\left(R_{R, 3}^{\mathrm{I}} R_{R, 1}^{\mathrm{II}}-R_{R, 3}^{\mathrm{II}} R_{R, 1}^{\mathrm{I}}\right) .
\end{aligned}
$$

Hence the effective data rates are:

$$
\begin{aligned}
U_{R}^{\mathrm{I}} & =\frac{\left(2 T-2 T^{\mathrm{I}}+T^{\mathrm{II}}\right) R_{R, 3}^{\mathrm{I}}}{2 T} \\
& =\frac{\left(R_{R, 1}^{\mathrm{I}} R_{R, 2}^{\mathrm{II}}+R_{R, 2}^{\mathrm{I}} R_{R, 1}^{\mathrm{II}}\right) R_{R, 3}^{\mathrm{I}}}{C_{b}}, \\
U_{R}^{\mathrm{II}} & =\frac{T^{\mathrm{II}} R_{R, 1}^{\mathrm{II}}}{2 T}=\frac{\left(R_{R, 3}^{\mathrm{I}} R_{R, 2}^{\mathrm{II}}+R_{R, 2}^{\mathrm{I}} R_{R, 3}^{\mathrm{II}}\right) R_{R, 1}^{\mathrm{II}}}{C_{b}} .
\end{aligned}
$$



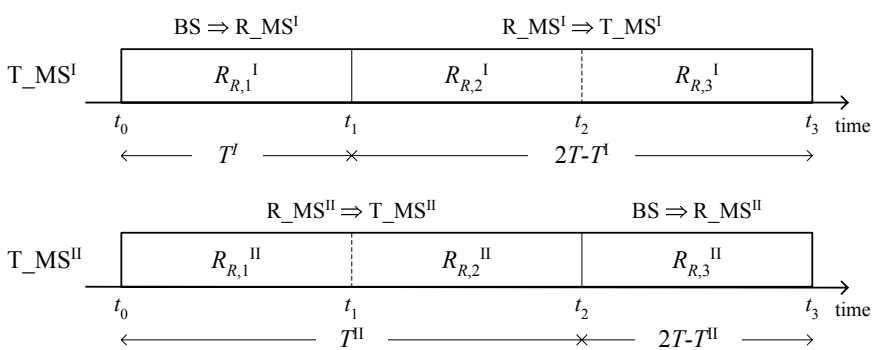

(a) Case: $T^{\mathrm{I}} \leq T^{\mathrm{II}}$
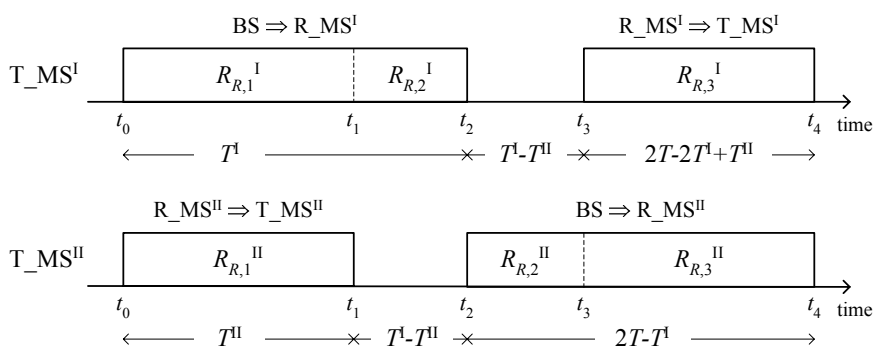

(b) Case: $T^{\mathrm{I}}>T^{\mathrm{II}}$

Fig. 3. Transmission timing for the type B concurrency.

The effective data rates for the direct link are given by $U_{D}^{\mathrm{I}} \equiv$ $R_{D}^{\mathrm{I}} / 2$ and $U_{D}^{\mathrm{II}} \equiv R_{D}^{\mathrm{II}} / 2$. Unlike in the case of the simple relaying and in the case of the type A concurrency, the effective data rate now should be calculated as half of the data rate of the link. This is because for a fair comparison with the type $B$ concurrency, the period assigned to the direct link should be regarded as $2 T{ }^{4}$

For the case of type B concurrency, we apply two different criteria for the selection of the multi-hop path. If the condition specified below is met, the multi-hop paths are selected for both target MS-s. Otherwise, the direct transmission is used.

- Criterion 1: If $U_{R}^{\mathrm{I}}+U_{R}^{\mathrm{II}}>U_{D}^{\mathrm{I}}+U_{D}^{\mathrm{II}}$, select the multi-hop paths.

- Criterion 2: If $U_{R}^{\mathrm{I}}>U_{D}^{\mathrm{I}}$ and $U_{R}^{\mathrm{II}}>U_{D}^{\mathrm{II}}$, select the multihop paths.

For both criteria, throughput gain can be represented by:

$$
G \equiv \begin{cases}\left(D^{\mathrm{I}}+D^{\mathrm{II}}\right) / 2 & \text { if the multi-hop paths are selected } \\ 1 & \text { otherwise }\end{cases}
$$

where $D^{\mathrm{I}} \equiv U_{R}^{\mathrm{I}} / U_{D}^{\mathrm{I}}$ and $D^{\mathrm{II}} \equiv U_{R}^{\mathrm{II}} / U_{D}^{\mathrm{II}}$.

\section{Performance Analysis}

We investigate here the impact of the type B concurrency on the downstream throughput. The system model for the performance evaluation is comprised of 19 cells. The hexagonal cell

\footnotetext{
${ }^{4}$ Recall that in the case of the type B concurrency both downstream channels share the whole period of $2 T$, but in the case of the direct transmission each downstream channel exploits relatively only half duration of $2 T$, i.e. $T$.
}

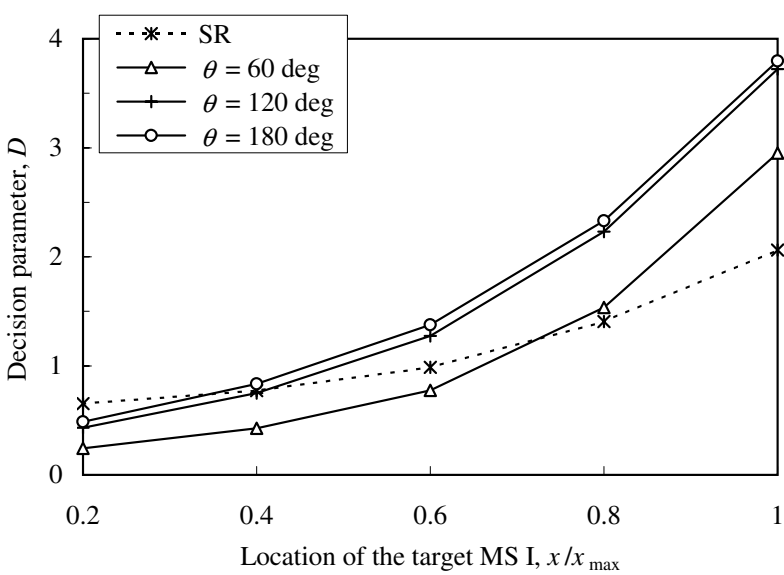

Fig. 4. the decision parameter for linear topology and no shadowing.

radius $x_{\text {max }}$ is $500 \mathrm{~m}$. The ratio of the transmission powers of a BS and a MS is set to 4, and the path loss exponent $\gamma$ is assumed to be 4 . The shadowing in $\mathrm{dB}, \zeta$, follows the Gaussian distribution with zero mean and standard deviation $\sigma=10 \mathrm{~dB}$. Both auto-correlation and cross-correlation are applied to the shadowing variables of links between a BS and an MS [1]. We also assume that the shadowing between MSto-MS links or between an MS-to-MS and a BS-to-MS links are all independent one from another.

A Monte Carlo computer simulation has been developed to evaluate the performance of the relaying schemes. For simplicity, the multi-hop relaying is employed only in the center cell. In the other cells, the direct transmission mode is assumed. The simulation collects statistical data from the center cell.

\section{A. Performance for Linear Topology}

In this section, we study the impact of the spatial separation of concurrent links, while varying the location of the two target MS-s. We place the target MS I at the given position $(x, y=$ $0)$ in Cartesian coordinates with respect to the center cell site, as shown in Fig. 2 (a). The target MS II is placed at $(x \cdot \cos \theta$, $-x \cdot \sin \theta$ ), so that the two MS-s are separated by the angle $\theta$. Each relaying MS is placed at the midway point between the BS and the target MS.

We fist consider this case without shadowing. Fig. 4 shows the value of $D$ for type $B$ concurrency for various locations of the target MS-s. The result for the simple relaying with 2-hop path is also shown in the figure and is denoted by $S R$. Note that by symmetry, $U_{R}^{\mathrm{I}}=U_{R}^{\mathrm{II}}$ and $U_{D}^{\mathrm{I}}=U_{D}^{\mathrm{II}}$, and so the criterion 1 and 2 result in the same decision. The results show that $D$ increases with the separation $\theta$, as expected. However, we can see that, in some cases, the concurrency may not provide a gain over simple relaying. In particular, when $\theta=60 \mathrm{deg}$, the simple relaying outperforms the concurrency except at the cell boundary. Hence, the results in this figure confirm that if the concurrent links are closed to each other, the concurrency should not be used. 


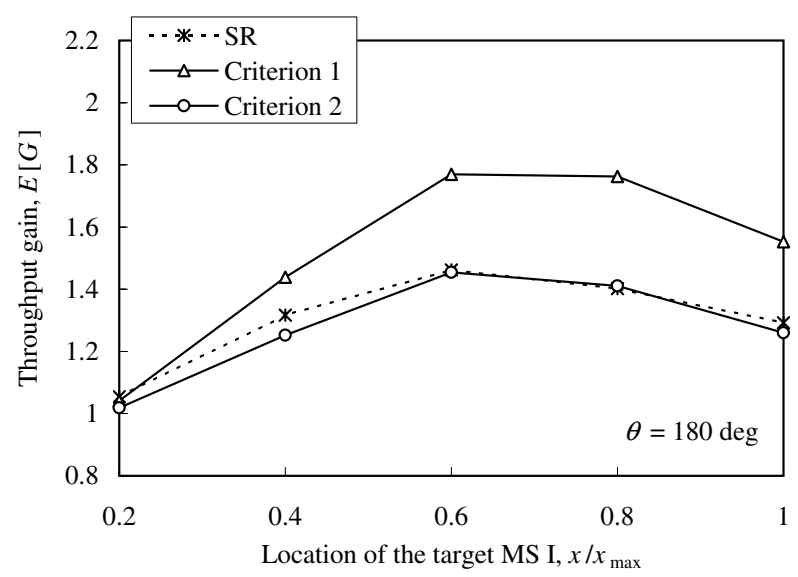

Fig. 5. The throughput gain for linear topology with shadowing.

We now continue our evaluation by incorporating shadowing into our system. Fig. 5 shows the results of $G$ for the shadowed environment. Note that $G$ is now a random variable due to the stochastic nature of the shadowing process, so we consider the expected value of $G, E[G]$. These results were calculated for $\theta=180 \mathrm{deg}$. We can see here that $E[G]$ can be increased by concurrency with the criterion 1 . However, since this criterion is based on the calculation of the total throughput, the throughput of one of the two target MS's throughputs may be lower than that with the conventional direct transmission, while the sum of the two throughputs is higher. In order to avoid the throughput decrease for any of the two target MS-s, the criterion 1 should be replaced by the criterion 2 . However, we note that, since the criterion 2 is more stringent in selecting the multi-hop path case, the performance of the criterion 2 is worse than that of the criterion 1, as shown in the figure. Nevertheless, the criterion 2 should be employed in order to ensure the superiority of the relaying system over the conventional system at any time.

The fact that the $E[G]$ with the criterion 2 is not higher than that with the simple relaying, as shown in Fig. 5, is due to the flexibility in the path selection in the case of the simple relaying. The type $B$ concurrency provides only two options such that, whether the multi-hop path or direct link is chosen, this choice is assigned to both target MS-s. On the other hand, with simple relaying, we can select and utilize the multi-hop path for each target MS individually. Such freedom of the path selection can lead to higher throughput gain than the concurrency.

From the the results above, one can expect that a hybrid scheme of the criterion 2 and the simple relaying will exploit both the concurrency and the flexible path selection. Besides, since the throughput improvement by concurrency is highly dependent on the separation of the concurrent links, the concurrency cannot always ensure a higher throughput gain than in the non-concurrent case. For a severe interference environment, the simple relaying would be a better choice. Therefore, we conclude that a hybrid scheme would provide better overall performance.

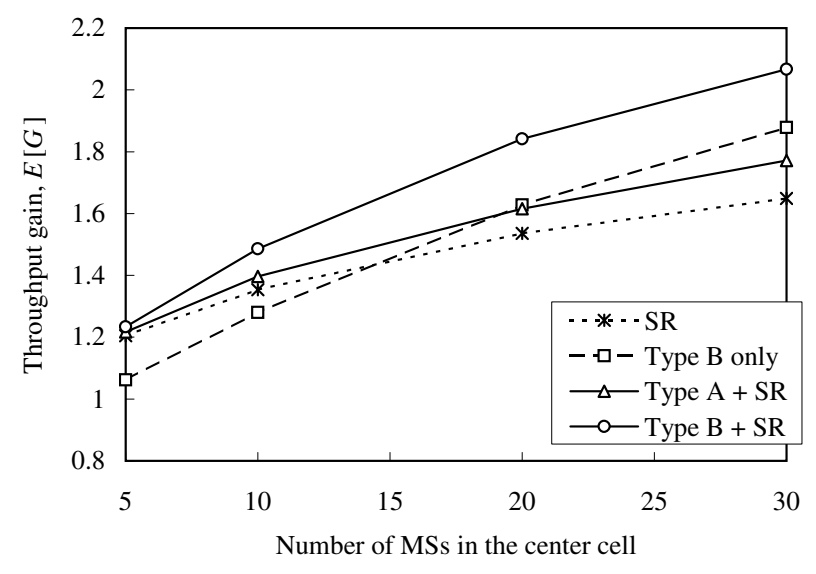

Fig. 6. Performance comparison of the concurrency in random topology.

Unlike the results in Fig. 4, in Fig. 5, we can see that $E[G]$ decreases at the cell boundary. This is due to the effect of the best BS selection. For detailed explanation of such this effect, the reader is referred to our previous work [1].

\section{B. Performance Comparison in Random Topology}

We present and compare the performances of various relaying schemes in random topology with shadowing. A number of MS-s which are capable of relaying and the target MS-s are assumed to be uniformly distributed over the cell area. We find the best path for each target MS, one with the maximum throughput.

In the hybrid control of the type $B$ concurrency and the simple relaying, we compare the three effective data rates for each target MS: of the direct single-hop link, of the multi-hop path with the simple relaying, and of the multi-hop path with the type B concurrency. Then we select the best scheme, the one with the maximum sum of the effective data rates for the two target MS-s. Considered the criterion 2 case, if any of the two effective data rates of the multi-hop paths with the type $B$ concurrency is smaller than that of the direct single-hop link, then type B concurrency is eliminated from the selection. Note that in this hybrid control scheme, the effective data rate with simple relaying should be considered for $2 T$, and then it is given by $1 /\left(2 \cdot\left(1 / R_{R, 1}+1 / R_{R, 2}\right)\right)$.

Fig. 6 shows $E[G]$ while varying the number of total MS-s in the center cell. The throughput for all the schemes increases with the number of MS-s, as expected. Compared to the simple relaying case, the stand-alone type $B$ concurrency does not provide a significant gain, similar to the results in the previous subsection. In particular, for the small number of MS-s, the performance of the type B concurrency is even worse than the simple relaying case. However, we observe that the throughput is considerably increased by the use of the proposed hybrid control scheme. The best scheme depends on the topology and may be either type B concurrency or the simple relaying, according to the MS locations and the shadowing. This comparison results support our claim that a hybrid scheme should be used in the general case. 
We also present the results for the hybrid control of the type A concurrency and simple relaying in Fig. 6 . The results were calculated for the case where the maximum number of allowable hops is 5. Note that except for this hybrid control, for three other cases in the figure the number of hops is limited to 2. The results in the figure show that the amount of increase offered by the type A concurrency is much smaller than that by the type $B$ concurrency. Since the concurrent transmissions in the type $B$ concurrency can be farther away from each other than in the type A concurrency, the increase in interference power can be smaller in the type $B$ concurrency case. From the results, we conclude that in order to achieve the best improvement, the concurrency should be applied between different downstream paths, rather than within the same downstream path.

\section{IMPACT ON THE CAPACITY OF THE CONVENTIONAL POWER-CONTROLLED SYSTEM}

We now discuss the impact of the multi-hop relaying on the capacity of the conventional CDMA system, where the power control is employed for providing a constant bit rate with the required quality, and the channel multiplexing within a cell is based on CDM (for downlink) or CDMA (for uplink).

Previous works showed that coverage of those systems can be increased by the multi-hop relaying, but it is not easy to enhance the capacity under the interference-limited conditions, such as the case in the high-loaded systems with small cells [3]-[7]. ${ }^{5}{ }^{6}$ This is mainly due to the interference increase from the concurrent relaying transmission. Recall that in the conventional systems, the near-far problem can be avoided by power control. However, in the multi-hop system, it is possible that the interfering transmitter, rather than the intended transmitter, is closer to the receiver. In such a case, any power control would be ineffective and would, in fact, result in very high interference level, possibly leading to total outage. ${ }^{7}$ Although such interfering transmission may be avoided by the use of a sophisticated algorithm for routing and time slot assignment, optimization of both selections of relaying MS and time slot seems to be difficult, because of the a large number of transmissions that would normally occur concurrently within the same cell.

In this work, we have realized the concurrency gain by allowing only two downstream paths at any time and also by utilizing both concurrent and the non-concurrent transmissions. Since the downstream paths are implemented in the

\footnotetext{
${ }^{5}$ Although Rouse et al. [7] evaluated the capacity improvement achieved through multi-hop relaying over the conventional system, such an improvement does not occur for all non-local traffic cases in which the source and the target are in different cells, or only one of the two resides in the wireless network. Note that in our paper the non-local traffic is assumed to be much more dominant than the local-traffic whose source and target are in the same cells.

${ }^{6}$ Zadeh et al. [10] demonstrated the capacity improvement achievable by multi-hop relaying, unlike in their previous work [4]. However, interference cancellation scheme such as multi-user detection (MUD) was employed in [10]. Note that in our work, we do not apply any scheme for the interference cancellation.

${ }^{7}$ Such near-far problem may also arise in mobile ad-hoc networks (MANETs) [11].
}

TDM fashion, in such a way that any period $2 T$ is dedicated to pair of MS-s, the path selection of the two MS-s does not affect the signal quality of the other MS-s in the same cell. So we have only to consider the interference between the two paths for these two MS-s. If this mutual interference is too high, it can be avoided by choosing the direct paths or the non-concurrent relaying paths. Clearly, such control will be easier in a TDM system than in a CDM system where the path selection for each MS affects one another. More comprehensive comparison of TDM and CDM in multihop system is left as a future study. Though, we can claim at least that the capacity gain by multi-hop relaying can be easier implemented in the rate-controlled CDMA system with TDM for multiplexing the same cell channels, than in the conventional power-controlled CDMA systems.

\section{CONCLUSIONS}

We have shown in this work that the downstream throughput can be increased by allowing concurrently relayed transmissions in the multi-hop cellular networks. However, the concurrency cannot always ensure a higher throughput gain than the non-concurrent system, because of the mutual interference between concurrently relaying links. In order to achieve a significant improvement, we should employ a hybrid control scheme utilizing both concurrency and non-concurrency. In addition, the concurrency should be applied among different downstream paths rather than between the hops on the same downstream path.

\section{REFERENCES}

[1] J. Cho and Z. J. Haas, "Throughput enhancement by multi-hop relaying in cellular radio networks with non-uniform traffic distribution," in Proc. IEEE VTC 2003 Fall, Orlando, FL, Oct. 2003

[2] S. Toumpis and A. Goldsmith, "Capacity regions for wireless ad hoc networks," in Proc. IEEE ICC 2002, New York City, NY, Apr. 2002, pp. 3168-3173

[3] A. Fujiwara et al., "Area coverage and capacity enhancement by multihop connection of CDMA cellular network," in Proc. IEEE VTC 2002 Fall, Vancouver, Canada, Sept. 2002, pp. 2371-2374

[4] A. N. Zadeh, and B. Jabbari, "Performance analysis of multihop packet CDMA cellular networks," in Proc. IEEE GLOBECOM 2001, San Antonio, TX, Nov. 2001, pp. 2875-2879

[5] T. J. Harrold and A. R. Nix, "Performance analysis of intelligent relaying in UTRA TDD," in Proc. IEEE VTC 2002 Fall, Vancouver, Canada, Sept. 2002, pp. $1374-1378$

[6] T. Rouse, S. McLaughlin, and H. Haas, "Coverage-capacity analysis of opprotunity driven multiple access (ODMA) in UTRA TDD," in Proc. IEE $3 G$ Mobile Communication Technologies, Mar. 2001, pp. 252-256

[7] T. Rouse, I. Band, and S. McLaughlin, "Capacity and power investigation of opprotunity driven multiple access (ODMA) networks in TDD-CDMA based systems," in Proc. IEEE ICC 2002, New York City, NY, Apr. 2002, pp. 3202-3206

[8] Q. Bi et al., "Performance of 1xEV-DO third-generation wireless highspeed data systems," Bell Labs Technical Journal, vol. 7, issue 3, pp. 97107, Mar. 2003

[9] A. Das et al., "Evolution of UMTS toward high-speed downlink packet access," Bell Labs Technical Journal, vol. 7, issue 3, pp. 47-68, Mar. 2003

[10] A. N. Zadeh, B. Jabbari, R. Pickholtz, and B. Vojcic, "Self-organizing packet radio ad hoc networks with overlay (SOPRANO)," IEEE Commun. Mag., vol. 40, no. 6, pp. 149-157, June 2002

[11] A. Muqattash and M. Krunz, "CDMA-based MAC protocol for wireless ad hoc networks," in Proc. ACM MobiHoc 2003, Annapolis, MD, pp. 153164, June 2003 